\title{
On-Demand Mobile Health Infrastructure for Remote Rhythm Monitoring within a Wait-and-See Strategy for Recent-Onset Atrial Fibrillation: TeleWAS-AF
}

\author{
Nikki A.H.A. Pluymaekers ${ }^{a} \quad$ Rachel M.J. van der Velden ${ }^{a} \quad$ Astrid N.L. Hermans $^{a}$ \\ Monika Gawalko ${ }^{a}$ Saskia Buskes ${ }^{a}$ Joyce J.H.M.W. Keijenberg ${ }^{a}$ Bianca Vorstermans ${ }^{a}$ \\ Harry J.G.M. Crijns ${ }^{a}$ Jeroen M. Hendriks ${ }^{c, d}$ Dominik Linz $^{a, b, d, e}$ \\ aDepartment of Cardiology, Maastricht University Medical Centre and Cardiovascular Research Institute, Maastricht, \\ The Netherlands; ${ }^{b}$ Department of Cardiology, Radboud University Medical Centre, Nijmegen, The Netherlands; \\ 'College of Nursing and Health Sciences, Flinders University, Adelaide, SA, Australia; ${ }^{\mathrm{d} C e n t r e ~ f o r ~ H e a r t ~ R h y t h m ~}$ \\ Disorders, University of Adelaide and Royal Adelaide Hospital, Adelaide, SA, Australia; 'Department of Biomedical \\ Sciences, Faculty of Health and Medical Sciences, University of Copenhagen, Copenhagen, Denmark
}

\section{Keywords}

Mobile health · Cardioversion · Atrial fibrillation ·

Wait-and-see strategy $\cdot$ Recent-onset atrial fibrillation

\begin{abstract}
Recently, we introduced the TeleCheck-AF approach, an ondemand mobile health (mHealth) infrastructure using appbased heart rate and rhythm monitoring for 7 days, to support long-term atrial fibrillation (AF) management through teleconsultation. Herein, we extend the mHealth approach to patients with recent-onset AF at the emergency department (ED). In the proposed TeleWAS-AF approach, on-demand heart rate and rhythm monitoring are used to support a wait-and-see strategy at the ED. All stable patients who present to the ED with recent-onset symptomatic AF and who are able to use mHealth solutions for heart rate and rhythm monitoring are eligible for this approach. Patients will receive both education on $\mathrm{AF}$ and instructions on the use of the mHealth technology before discharge from the ED. A case coordinator will subsequently check whether patients
\end{abstract}

are able to activate the mHealth solution and to perform heart rate and rhythm measurements. Forty hours after AF onset, the first assessment teleconsultation with the physician will take place, determining the need for delayed cardioversion. After maximal 7 days of remote monitoring, a second assessment teleconsultation may occur, in which the rhythm can be reassessed and further treatment strategy can be discussed with the patients. This on-demand mHealth prescription increases patient involvement in the care process and treatment decision-making by encouraging selfmanagement, while avoiding excess data-load requiring work-intensive and expensive data management. Implementation of the TeleWAS-AF approach may facilitate the management of AF in the ED and reduce the burden on the ED system, which enhances the capacity for health care utilization.

(c) 2021 S. Karger AG, Base

Nikki A.H.A. Pluymaekers, Rachel M.J. van der Velden, Astrid N.L. Hermans: Shared first authorship.

Jeroen M. Hendriks and Dominik Linz: Shared last authorship. karger@karger.com

www.karger.com/crd

Karger ${ }^{\prime}=$ (c) 2021 S. Karger AG, Base

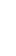

Dominik Linz

Maastricht Heart and Vascular Center

Maastricht University Medical Center

NL-6202 Maastricht (The Netherlands)

dominik.linz@mumc.nl 


\section{Introduction}

Several mobile health (mHealth) solutions have been recently introduced to screen for and to support management of atrial fibrillation (AF) [1]. In response to cancellations of outpatient services and conversion into teleconsultations as part of the COVID-19 restrictions, we developed the TeleCheck-AF approach, which is an ondemand mHealth infrastructure incorporating an appbased heart rate and rhythm monitoring to support remote AF management through teleconsultation. The approach has been described in detail elsewhere $[2,3]$.

Based on the feasibility of the TeleCheck-AF approach [2], we aim to extend the mHealth approach to the management of recent-onset AF in the emergency department (ED). Patients with recent-onset AF commonly undergo immediate restoration of sinus rhythm (SR) by pharmacologic or electrical cardioversion (CV). However, the Rate Control vs. Electrical Cardioversion Trial 7 - Acute Cardioversion vs. Wait and See (RACE 7 ACWAS) study showed that a wait-and-see (WAS) strategy in patients with recent-onset $\mathrm{AF}$ (rate control for symptom relief followed by delayed CV if needed $<48 \mathrm{~h}$ ) allows for spontaneous conversion to SR in $69 \%$ of patients, obviating active $\mathrm{CV}[4]$. Recurrences within 1 month were seen in $30 \%$ of patients in both groups, that is, the initially chosen strategy did not affect the recurrence pattern [4]. The WAS approach is now also mentioned as an option to manage patients with recent-onset AF in the current ESC Guidelines for the diagnosis and management of AF [5]. However, a clear practical guidance for this WAS approach and advice on how to perform rhythm monitoring are not provided. In this article, we describe the implementation of the TeleWAS-AF approach, an on-demand mHealth-based heart rate and rhythm monitoring infrastructure to support a WAS strategy for patients presenting with recent-onset $\mathrm{AF}$ at the $\mathrm{ED}$.

\section{Methods/Design}

\section{Implementation of TeleWAS-AF in an ED}

An overview of the TeleWAS-AF approach, the different steps during the decision-making process, and the subsequent assessment teleconsultations is summarized in Figure 1.

Who Is Eligible for a WAS Strategy Supported by

TeleWAS-AF?

All patients who present to the ED with an episode of recentonset ( $<36 \mathrm{~h}$ ) symptomatic AF, documented on an electrocardiogram (ECG) without signs of acute heart failure, acute myocardial infarction, or hemodynamic instability, and who are able to oper-

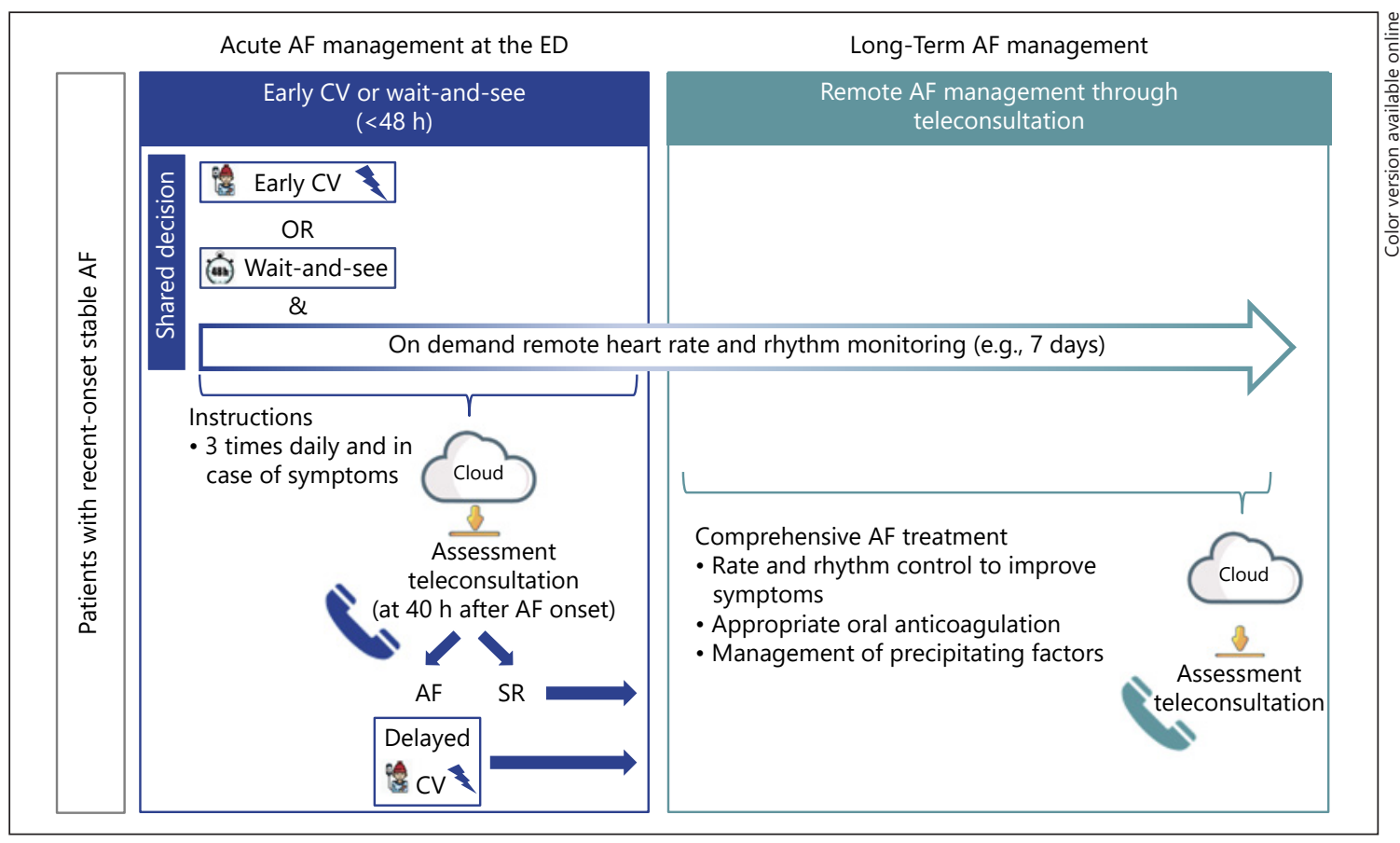

Fig. 1. Overview of the TeleWAS-AF approach, the different steps during the decision-making process, and the subsequent assessment teleconsultations. ED, emergency department; CV, cardioversion; AF, atrial fibrillation; SR, sinus rhythm; WAS, wait and see.

mHealth for Remote Rhythm Monitoring within a Wait-and-See Strategy 


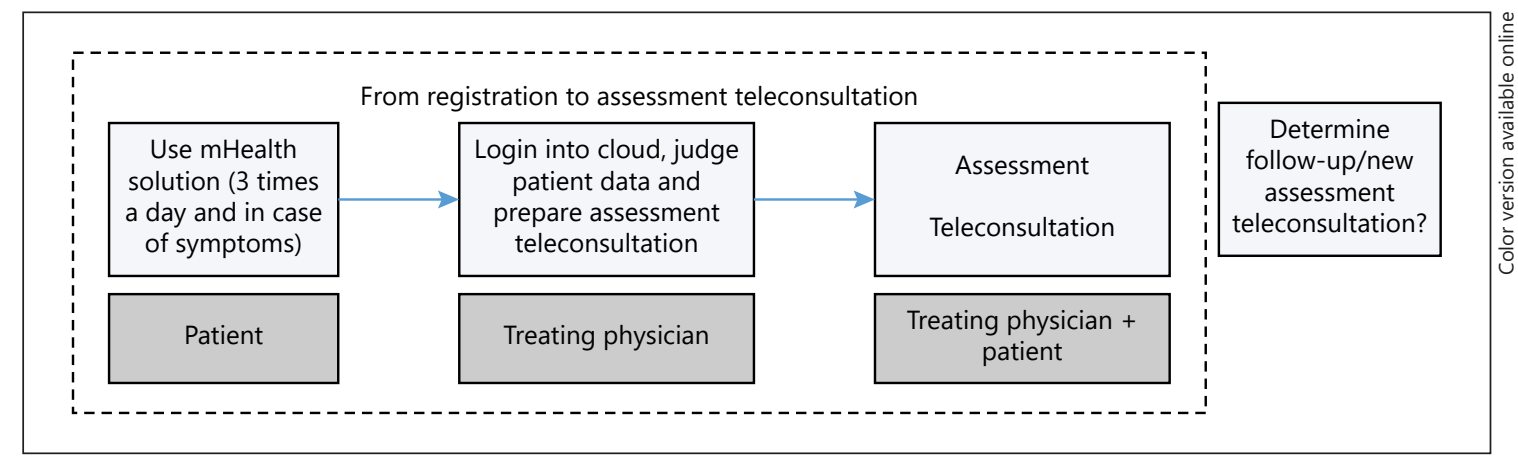

Fig. 2. Organization of the care pathway. Patients were asked to measure their heart rates and symptoms by the mHealth solution 3 times a day and in case of symptoms. Before the assessment teleconsultation, the treating physician logs into the cloud to evaluate the measurement. mHealth, mobile health.

ate mHealth solutions for heart rate and rhythm monitoring and own a smartphone are eligible to use the TeleWAS-AF approach. There are two scenarios how an eligible patient may present to the ED: (1) patients with recent-onset AF who directly present to the ED with symptomatic AF. or (2) a general practitioner or a medical specialist refers a patient with a recent-onset AF episode to the ED.

Patient Education and Instruction at the ED

Instruction and education of the remote on-demand heart rate and rhythm monitoring to patients are crucial for successful implementation of the mHealth approach. The multidisciplinary ED team, including nurses, a case coordinator, and physicians, has an important role in administering clear guidance to the patients about why, how, and when to use the mHealth solution for heart rate and rhythm monitoring.

\section{Patient Education}

Patients will be educated about AF, AF-related symptoms, possible complications, and potential treatment options, specifically the acute treatment of AF, for example, the WAS strategy or early CV. In almost $70 \%$ of the cases [3], the heart rhythm spontaneously recovers to SR and immediate CV is not needed (and therefore possibly resulting in the avoidance patients' overtreatment). In the case of the WAS strategy, heart rate lowering medication (such as $\beta$-blocker, verapamil, and digoxin) can be prescribed to reduce complaints of $\mathrm{AF}$, and afterward, the patients can await spontaneous conversion to SR at home. To monitor the heart rate and rhythm during the WAS period, an mHealth solution, such as handheld devices (e.g., AliveCor and MyDiagnostick) or mobile apps (e.g., FibriCheck), connected to a cloud can be used for maximal 7 days after the ED visit (7-day mHealth-prescription) [1]. In our initial pilot studies, we used the FibriCheck app using the photoplethysmogram (PPG) technology through the smartphone's built-in camera, which can be downloaded on the patients' smartphone $[2,3]$.

\section{Patient Instruction}

Why: The ED nurse explains that the mHealth solution is used for remote heart rate and rhythm assessment during the waiting period of the WAS strategy. Therefore, a 7-day mHealth-prescription to use a handheld device or an app is provided.
How: The ED nurse gives instructions to the patients on how to use the handheld device or install the app on the smartphone and a patient manual.

When: The provided patient manual instructs the patients to perform 3 measurements of heart rate and rhythm a day (morning, afternoon, and evening) and additional measurements in case of symptoms during days until the teleconsultation with the treating physician.

Implementation of Heart Rate and Rhythm Information into the Assessment Teleconsultations

The case coordinator checks the cloud if patients are able to activate the handheld device or the app and to perform heart rate and rhythm measurements. In case patients need further support, the case coordinator can be contacted. The telephone number of the ED for urgent matters is provided in the patient manual. All healthcare professionals have access to the raw rhythm traces of their patients via the cloud. Prior to teleconsultation, the physician logs into the cloud and can assess heart rate, rhythm, and symptoms recorded by the patients. The flowchart of the assessment teleconsultations is provided in Figure 2.

First assessment teleconsultation at $40 \mathrm{~h}$ after $\mathrm{AF}$ onset: If the telemonitoring recordings suggest SR, no further action is required, and patients will continue their heart rhythm and rate recordings. If AF remains to be present, a $\mathrm{CV}$ may be performed within $48 \mathrm{~h}$ after AF onset.

Second assessment teleconsultation at 7 days: After maximal 7 days of the mHealth prescription, the handheld device or the app expires, and the data collection stops. If the recordings suggest SR, no further action is required. If $\mathrm{AF}$ remains to be present or $\mathrm{AF}$ recurrence occurs, the subsequent strategy can be further discussed together with the patients. A CV can be performed. Alternatively, the pharmacological rate and/or rhythm control can be optimized including AF catheter ablation. In this case, patients are asked to repeat the measurements for one more week to check if medication changes for rate and rhythm control were effective or not. For this, they receive a new mHealth prescription which then can be activated, and a follow-up assessment teleconsultation will be planned.

According to the current ESC AF guidelines, the following points need to be considered in regard to a WAS strategy [5]. (1) In anticoagulated AF patients, a WAS strategy can be applied, 
irrespective of duration of the current AF-episode. (2) In patients with recent-onset $\mathrm{AF}$ with a definite duration of $\mathrm{AF}$ $<48 \mathrm{~h}$, an early CV can be performed, irrespective of anticoagulation. (3) If a CV is performed in hemodynamically stable patients with symptomatic AF, the choice between electrical and pharmacological CV should be guided by patient and physician preferences aligned with guideline recommendations. (4) In patients at risk for stroke, anticoagulant therapy should be continued long term after CV according to the long-term anticoagulation recommendations, irrespective of the method of $\mathrm{CV}$ or the apparent maintenance of SR. In patients without stroke risk factors, anticoagulation is still under debate and is recommended for 4 weeks after CV by some societies (e.g., the Canadian Cardiovascular Society [6]). (5) If a CV is intended in patients with $>48$ h recent-onset AF without anticoagulation, a transesophageal echocardiography to exclude an atrial thrombus or effective anticoagulation for a minimum of 3 weeks is recommended. The safety of early compared to late $\mathrm{CV}$ is discussed in more detail in a recent review by Khatami et al. [7].

\section{Discussion}

There are several points which make the TeleWAS-AF approach unique, for example, experiences within integrated AF care and active patient involvement in the care process as well as in the treatment decision is crucial. In fact, patients take responsibility of their care, and healthcare professionals rely on the self-care of patients (i.e., measuring heart rate and rhythm and reporting on experienced symptoms). Therefore, it is of significant importance to prepare patients for such role, by means of 3 Es: engagement: identify the patients' needs, values, and preferences; provide clear education and instruction in a way that patients understand what is expected from them; and encourage them to undertake self-management (i.e., measuring vital parameters) and involve them in informed or shared decision-making regarding their AF treatment. This novel approach may require significant changes and redesign in the delivery of care [8].

Heart rate and rhythm monitoring in the TeleWAS$\mathrm{AF}$ approach can be performed by handheld devices or app-based mHealth solutions using the ECG or PPG technology. In general, the PPG technology has limitations in diagnosing $\mathrm{AF}$ as current $\mathrm{AF}$ guidelines require an ECG documentation of an AF episode $[5,6]$. TeleWAS-AF is exclusively used in patients who present with recent-onset AF and get a documentation of the AF episode at the ED. Therefore, the PPG-based app is not used for the diagnosis of AF but for the heart rate and rhythm assessment within the WAS strategy.

The limited validity for maximal 7 days, regulated by a 7-day mHealth prescription, avoids unnecessary data load requiring work-intensive and expensive data management. If an app-based mHealth solution is used, no hardware is required, which has several hygienic and logistical advantages and makes this TeleWAS-AF approach available at low costs.

How many patients could profit from the TeleWasAF approach? First results from the TeleCheck-AF project showed that age is not an exclusion criteria since overall $80 \%$ of patients were willing and able to participate in the TeleCheck-AF approach [9]. Further, based on our experience from the RACE 7 ACWAS study [4], we expect that one-third of all patients presenting with recent-onset $\mathrm{AF}$ at the ED may be eligible for TeleWASAF. In a subgroup of patients included in the TeleCheckAF project [2,3], we have already successfully applied the TeleWAS-AF approach in $>200$ patients presenting with recent onset AF. Remote rate and rhythm monitoring by TeleWAS-AF was feasible, and feedbacks from patients were positive.

\section{Conclusions and Future Implications}

Herein we describe a new mHealth approach facilitating acute management of recent-onset AF by a WAS strategy, which will impact the management of AF in the ED system. While maintaining the delivery of comprehensive treatment, the approach has a potential to reduce the burden on EDs and enhance the capacity for healthcare utilization. However, given the change in the treatment approach, redesign of care delivery and reimbursement models may be warranted. Obviously, patients' role in the decision-making process and the communication between patients and the treatment team is crucial. This approach will be implemented in all participating centers of the TeleCheck-AF project [3]. Long-term, we are currently conducting a multicenter randomized clinical trial RACE 9 OBSERVE AF (Reg number NL73104.068.20) in which a device-based watchful waiting strategy, that is, symptom reduction through rate control medication and monitoring for 4 weeks until spontaneous conversion is achieved compared to standard care, consisting of either early or delayed CV according to the TeleWAS-AF approach.

\section{Conflict of Interest Statement}

The authors have no conflicts of interest to declare. 


\section{Funding Sources}

The authors declare that they did not receive any funds for this study.

\section{Author Contributions}

All authors were involved in the development of the TeleWASAF strategy and contributed to the writing process of the manuscript.

\section{References}

1 Hermans ANL, van der Velden RMJ, Gawalko M, Verhaert DVM, Desteghe L, Duncker $\mathrm{D}$, et al. On-demand mobile health infrastructures to allow comprehensive remote atrial fibrillation and risk factor management through teleconsultation. Clin Cardiol. 2020 Nov;43(11):1232-9.

2 Pluymaekers NAHA, Hermans ANL, van der Velden RMJ, Gawałko M, den Uijl DW, Buskes $\mathrm{S}$, et al. Implementation of an on-demand app-based rate and rhythm monitoring infrastructure for the management of atrial fibrillation through teleconsultation: TeleCheckAF. Europace. 2020:euaa201.

3 Linz D, Pluymaekers NAHA, Hendriks JM. TeleCheck-AF for COVID-19. Eur Heart J. 2020 Jun 1;41(21):1954-5.
4 Pluymaekers NAHA, Dudink EAMP, Luermans JGLM, Meeder JG, Lenderink T, Widdershoven J, et al. Early or delayed cardioversion in recent-onset atrial fibrillation. $\mathrm{N}$ Engl J Med. 2019 Apr 18;380(16):1499-508.

5 Hindricks G, Potpara T, Dagres N, Arbelo E, Bax JJ, Blomström-Lundqvist C, et al. 2020 ESC Guidelines for the diagnosis and management of atrial fibrillation developed in collaboration with the European Association of Cardio-Thoracic Surgery (EACTS). Eur Heart J. 2021 Feb 1;42(5):373-498.

6 Andrade JG, Verma A, Mitchell LB, Parkash R, Leblanc K, Atzema C, et al. 2018 focused update of the canadian cardiovascular society guidelines for the management of atrial fibrillation. Can J Cardiol. 2018 Nov;34(11):137192.
7 Khatami M, Pope MK, Le Page S, Radic P, Schirripa V, Grundvold I, et al. Cardioversion safety: are we doing enough? Cardiology. 2020;145(11):740-5.

8 van der Velden RMJ, Hermans ANL, Pluymaekers NAHA, Gawałko M, Vorstermans B, Martens $\mathrm{H}$, et al. Coordination of a remote mHealth Infrastructure for atrial fibrillation management during COVID-19 and beyond: TeleCheck-AF. Int J Care Coord. 2020.

9 Pluymaekers NAHA, Hermans ANL, van der Velden RMJ, den Uijl DW, Vorstermans B, Buskes S, et al. On-demand app-based rate and rhythm monitoring to manage atrial fibrillation through teleconsultations during COVID-19. Int J Cardiol Heart Vasc. 2020 May;28:100533. 